\title{
La simplificación en el mensaje periodístico. La dinámica dualista: el caso Assange
}

institucionales.us.es/ambitos/

\section{Helena Ramírez Barragán}

Universidad de Sevilla

helenramirezbarragan@gmail.com

English Version: The simplification in the journalistic message. The dualistic dynamic: the Assange case

En este artículo, fruto de una investigación académica con motivo de la realización de un Trabajo de Fin de Grado, buscamos definir el concepto de simplificación del mensaje. La búsqueda de esta definición se lleva a cabo basándonos en varios autores provenientes del mundo de la Estructura de la Información y la Psicología Social de la Comunicación. Dada la amplitud del concepto, hemos delimitado la investigación ofreciendo una visión más básica y fundamental de los aspectos

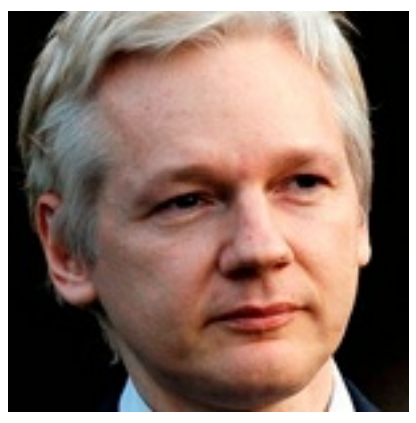
tratados. A lo largo del trabajo, hemos tratado de establecer cuáles son aquellas características que cumple lo que podríamos denominar la prensa contemporánea, que nunca había vivido con tanta prisa, y que queda tan ligada a los tejemanejes del Poder. Para ello, hemos recurrido a autores elementales de la Comunicación, como Ramón Reig y otros investigadores, aunque también se ha buscado introducir, de forma reducida y lacónica, una explicación neurocientífica para así dar respuestas al perfecto funcionamiento del "periodismo simple".

Palabras clave: EEUU, poder, telaraña mediática, manipulación, opinión pública, periodismo, héroe, terrorista, ataque militar, hackers.

Abstract: In this article, the result of an academic research on the completion of aFinal Degree Project, we seek to define the concept of simplification of the message. The search for this definition is carried out based on several authors from the world of the Information Structure and the Social Psychology of Communication. Given the breadth of the concept, we have delimited the research offering a more basic and fundamental view of the aspects treated. Throughout the work, we have tried to establish which are those characteristics that meets what we could call the contemporary press, which had never lived with such haste, and which is so linked to the machinations of Power. For this, we have turned to elementary authors of the Communication, such as Ramón Reig and other researchers, although we have also sought to introduce, albeit in a reduced and laconic way, a neuroscientific explanation to give answers to the perfect functioning of "simple journalism".

Keywords: USA, power, media cobweb, manipulation, public opinion, journalism, hero, terrorist, military attack, hackers. 


\section{Introducción}

Y luego habla, o ladra, de un modo complicado. Nosotros aullábamos y por

imitarle aprendimos a hablar, y ni aún así nos entendemos con él. Solo le entendemos de veras cuando él aúlla. Cuando el hombre aúlla, grita o amenaza le entendemos muy bien los demás animales. ¡Cómo que entonces no está distraído en otro mundo!... Pero ladra a su manera, habla, y eso le ha servido para inventar lo que no hay y no fijarse en lo que hay. En cuanto le ha puesto un nombre a algo, ya no ve este algo, no hace sino oír el nombre que le puso, o verle escrito. La lengua le sirve para mentir, inventar lo que no hay y confundirse. Y todo es en el pretexto para hablar con los demás o consigo mismo. Y hasta nos ha contagiado a los perros. (Miguel de Unamuno, Niebla 1907).

Niebla es la obra magna del afamado escritor Miguel de Unamuno que este escribió en 1907 y publicó en 1914. En ella se narra la vida de un joven llamado Augusto Pérez que convive con una crisis existencial de tal tamaño que le hace cuestionarse cada paso de sus cotidianas andanzas. Al final del relato, Augusto visita a Unamuno, quien le afirma que no es más que un ente de ficción creado por él para su Nivola. Podríamos decir que la vida de Augusto Pérez y lo que le concierne es tan real como Unamuno, y la pluma con la que escribiera, quiso que fuere.

Este párrafo son las palabras del perrito de Augusto, su gran amigo fiel, aparentemente insulso en inteligencia y capacidad reflexiva, que oye cada día los monólogos existencialistas de su dueño. Una vez fallecido Augusto, es a su perrito Orfeo a quien le toca reflexionar sobre la vida humana. Según sus pensamientos, el ser humano es un animal que al hablar y comunicarse del modo en que lo hace, deja de serlo, o al menos, deja de ser inteligible por el resto de la fauna, y apunta que la principal razón es porque cuando hablamos inventamos una realidad que no hay, lo cual nos impide ver lo que realmente tenemos delante: "En cuanto le ha puesto un nombre a algo, ya no ve este algo, no hace sino oír el nombre que le puso, o verle escrito." Esto significa que hemos inventado algo que nos distingue, y que, sin embargo, lo usamos para mentir, y mentirnos.

Esas palabras, de Orfeo o Unamuno, según se prefiera, pueden ser aplicadas a la realidad vigente del periodismo. Vivimos en un mundo cuya realidad social es construida por el periodismo, y en ocasiones incluso creadas por u originadas desde él mismo, y todo lo que este conlleva: desde el periodista que teclea las letras en su ordenador de la redacción, hasta el lector que sostiene con sus manos el periódico. O podemos ir más lejos y profundizar en el espeso velo de humo que oculta tanta telaraña (Reig: 2010), desde el magnate multimillonario, al oyente que escucha el telediario de fondo: es decir, de los dueños del periodismo a los esclavos del mismo.

Para comprender, entonces, el comportamiento que ha venido teniendo el periódico El País con, probablemente, el hacker más conocido de la historia, es necesario sentar las bases de varios conceptos de algunos autores de referencia para esta investigación.

Ramón Reig, catedrático de Estructura de Información por la Universidad de Sevilla, describe a ese entramado de poder de índole socioeconómico como una "telaraña mediática", ya que actúa, directa o indirectamente, sobre la información que el público 
recibe, aunque dichos poderes provengan de sectores para nada relacionados con el mundo de la comunicación. Esto unido al "espejismo digital" del que habla Rendueles en su ensayo Sociofobia nos va recordando a ese lenguaje de mentiras o realidades creadas al que hacía referencia Orfeo, el perrito de Augusto, cuando observamos ese feedback creado entre la información generada por el periódico en cuestión (la supuesta realidad contada -y compartida-) y la respuesta gestada sobre los individuos consumidores del mismo, pues no olvidemos, además, según la Teoría de Sociedad de Luhmann, que no son los individuos sino las comunicaciones las unidades constituyentes y reproductoras de los sistemas sociales, siendo la comunicación, entonces, el verdadero punto de partida.

Todos estos aspectos y características de la sociedad y la comunicación actual es lo que sostiene lo que hemos denominado 'El Caso Assange', y es también aquello que ha marcado al australiano primero como héroe, luego como villano, y hoy como un personaje olvidado. Pero sobretodo, es la maquinaria que ha conseguido que sea esa y no otra la realidad aceptada (y vivida) por y para las masas.

\section{Objetivos e hipótesis}

A) Objetivos:

- Estudiar las distintas coberturas temporales ofrecidas por El País sobre el caso Assange.

- Demostrar que ha habido un antes y un después en dicha cobertura que caracterizan a Assange primero como héroe luego como villano.

- Ofrecer una explicación fundamentada en las ciencias sociales (como la Psicología Social de la Comunicación o la Economía Política de la Información, la Comunicación y la Cultura) que responda a tal cobertura.

B) Hipótesis:

- Julian Assange al principio fue un personaje querido por El País y luego alguien nocivo para la sociedad según dicho periódico.

- Mientras Julian Assange fue concebido como bueno, se le atribuía vocablos con connotación positiva como "periodista" o "ciberactivista".

- El antes y el después lo marca, no solo EEUU, sino el hecho de que Ecuador ofreciera asilo a Julian Assange.

\section{El País en la Estructura}

Para realizar la investigación relativa al caso Assange hemos analizado los artículos pertenecientes al periódico español El País. La razón por la que hemos escogido tal medio de comunicación es por la involucración que tuvo en el momento cablegate y sus posteriores consecuencias, hasta llegar a la actualidad que rodea al hacker australiano.

Grosso modo, El País se autodefine a sí mismo como un diario independiente en el párrafo 1 del primero de sus artículos editoriales:

1.1. EL PAís se define estatutariamente como un periódico independiente, nacional, de información general, con una clara vocación de europeo, defensor de la democracia pluralista según los principios liberales y sociales, y que se compromete a guardar el orden 
democrático y legal establecido en la Constitución.

Pero el periódico, aunque autodeclarado independiente en el primero de sus principios editoriales, también tiene dueños con nombres y apellidos, y por tanto, límites en sus escritos, no olvidemos que ya sufrió acusaciones de censura:

\section{Más de 20 colaboradores de El País denuncian la censura en el periódico}

La censura y el clima de amenazas e intimidación que la dirección de El País ha impuesto en la redacción ha terminado por rebotarles en la cara, en forma de sonora y pública bofetada, a Juan Luis Cebrián, presidente de Prisa, y al director, Javier Moreno.
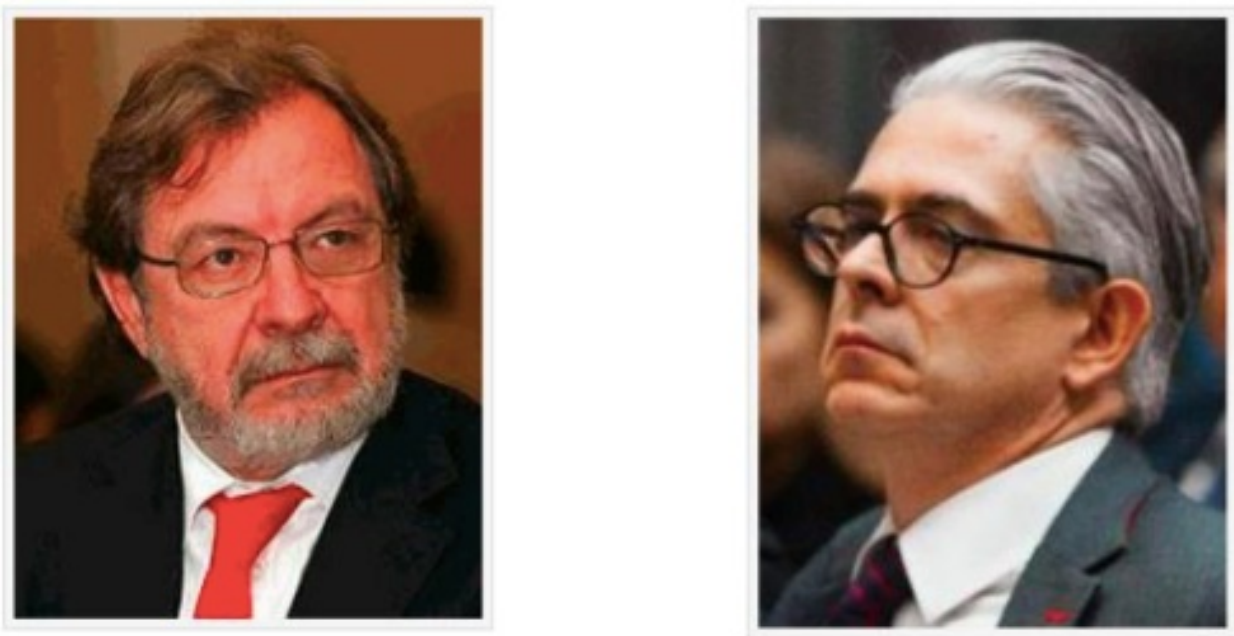

Juan Luis Cebrián, a la izquierda, y Javier Moreno.

Imagen 1. La censura en El País.

Fuente: Ere-EIPais.com

\subsection{Algunos apuntes sobre PRISA}

De todos es sabido que como miembro del gran grupo Prisa, hijo predilecto de Polanco y Spottorno, rico empresario uno y rico ingeniero el otro, responde y acata las órdenes de un poder que siempre tratará de defenderse contra aquello que se rebele, en este caso, Julian Assange.

PRISA es el mayor de los grupos de comunicación de este país y el primero en el mercado de lengua española y portuguesa, siendo líder en materia de información, educación y entretenimiento, y estando presente en 22 países.

El grupo fue fundado en 1958 por la Editorial Santillana a manos de Jesús Polanco. En los 70 comienza con El País hasta que poco a poco se crece como conglomerado, siendo hoy propietario de Cadena Ser, Cinco Días, Diario AS y Los 40 Principales, entre otros. Se considera que El País es de las propiedades más importantes del grupo. 


\section{Delimitando el concepto de simplificación en el mensaje}

En este apartado llevaremos a cabo la tarea de aplicar la Psicología Social de la Comunicación y la Teoría de la Propaganda al caso Assange, siguiendo el trabajo de diversos profesionales de estas especializaciones. Esto nos ayudará en nuestro objetivo de dar respuesta a por qué la opinión pública se comporta como lo hace en casos tan segmentarios como este.

\subsection{Julian Assange, el Héroe del momento}

Las psicólogas Trinidad Núñez Domínguez y Susana Bayó Berenguer en el capítulo 7 del libro Competencias sociales para profesionales de los medios, dedicado a la persuasiónmediática, resumen el resultado de un estudio realizado al periódico TheGuardian cuando este cubrió la guerra del Golfo (1990-1991), durante el mandato de George Bush "padre" (1989-1993). El resultado sirvió de base en dicho libro para ofrecer una definiciónpráctica de los conceptos relativos a la propaganda.

En relación con este estudio, para el que se aplicó una tabla comparativa entre el bando aliado y el bando enemigo, así como otra relativa a las figuras representativas [George Bush (bueno) versus Sadam Husein (malo)], hemos resuelto nuestra propia tabla de valores, en términos lingüísticos, para mostrar el trato recibido por Julian Assange durante su momento de gloria, en este caso en el periódicoespañolEl País.

\begin{tabular}{|c|c|}
\hline $\begin{array}{c}\text { Actores políticos y Empresas: } \\
\text { Son villanos }\end{array}$ & $\begin{array}{c}\text { Julian Assange: } \\
\text { Es un héroe poco } \\
\text { Apoyado }\end{array}$ \\
\hline Fuerzan & Es forzado \\
\hline Obligan & Es obligado \\
\hline Persiguen & Es perseguido \\
\hline Silencian & Es silenciado \\
\hline Marginan & Es marginado \\
\hline Deshabilitan & Es deshabilitado (en Internet, medios...) \\
\hline Conspiran & Conspiran contra él, pero él no conspira \\
\hline
\end{tabular}

Tabla 1. Tabla comparativa de valores

Fuente: Elaboración propia.

Siguiendo con el análisis de las psicólogas ya nombradas, "las personas que reciben el impacto propagandístico pueden responder o no a él. En el caso de que sí respondan, pueden hacerlo manteniendo una de estas tres actitudes: Conformismo; Convicción; Conversión".

Esto significa que los lectores de la información publicada por el periódico El País durante, al menos, ese periodo de cobertura mediático analizado, han podido:

a) Conformarse con la idea producida de Julian Assange como héroe de la sociedad, que filtró información clasificada por el bien humanitario. Es decir, este paso requiere un nivel de esfuerzo interno muy bajo o casi nulo, pues el lector sencillamente acepta el mensaje 
que le llega como cierto, y no se propone acto de reflexión alguno a fin de verificar la certeza del mismo.

b) Se convence de lo emitido por el periódico. En este, llamémosle, nivel de recepción, el lector queda tan convencido de que lo que lee es cierto que predica con el ejemplo y termina actuando conforme lo que ha leído en el medio. Los receptores aquí podrían involucrarse en tareas sencillas como, por ejemplo, servir de apoyo a Julian Assange y Wikileaks en tertulias foreras, tuits, comentarios en Facebook o versiones digitales de periódicos que traten noticias al respecto, etc.

c) En cuanto a la conversión de la sociedad, se trata de multiplicar el efecto recaída sobre ella en el caso anterior, pues se habla, entonces, de una sociedad convencida. Digamos, pues, personas más comprometidas en sus actos con el caso que fuere, en este caso, con el ideal de considerar a Julian Assange como un héroe que lucha por el bien mundial contra la superpotencia estadounidense. Aquí, los lectores podrían servir de apoyo a Assange y su plataforma de filtraciones dando un paso más que el anterior, por ejemplo, donando dinero a Wikileaks o acudiendo a manifestaciones por la libertad del, denominémosle, herético cibernético.

Núñez Domínguez y Bayó Belenguer hablan entonces de las cinco reglas de la propaganda: Simplificación- Exageración - Orquestación - Transferencia - Contagio.

Es decir, se ofrece un mensaje "breve y claro" exagerándolo, "orquestando ese mensaje para que multiplique su efecto" (p. 126), que será transferido a un público que, a su vez, lo contagiará a su contexto. Añaden las expertas que es este último paso aquel con el que se consigue la unanimidad perseguida.

Ahora la cuestión sería cómo es que El País, pieza más de la telaraña mediática (Reig:2010) y herramienta del poder, pudo publicar esas informaciones que pintaban al hacker más temido por el pentágono en un héroe de merecidos honores. Pues sírvase la aclaración de Ramón Reig sobre la revista Time en su libro Los dueños del periodismo (2011) y apliquémosla al periódico español:

Time juega a la libertad de expresión y se da un baño de progresismo en relación con sus lectores. Se trata de vender, no de informar de manera sincrónica y estructural. Este tipo de mensajes es una de las relativas excepciones a la norma.

\subsection{Julian Assange convertido en villano después de pedir asilo a Ecuador}

Apuntábamos entre nuestras hipótesis que El País pegaba el volantazo ideológico allá por el 2012, cuando el refugiado sin refugio, Julian Assange, pedía asilo a Ecuador.

En 2012 la propaganda favorecedora hacia Julian Assange se cambia de atuendos y modifica sus palabras positivas dedicadas al herético para dirigirlas al antiguo héroe, EEUU, y viceversa, las malas definiciones pasan de EEUU a Assange.

Para llevar a cabo esta segunda parte del estudio hemos escogido una batería de aproximadamente una decena de documentos, entre los cuales encontramos noticias, crónicas y análisis. La razón por la que finalmente tomamos la decisión de profundizar 
también en los análisis y no solo limitarnos a las noticias, es que consideramos que son el corazón del periódico y que demuestran considerablemente cuál es el posicionamiento del mismo.

Ofreciendo una muy breve síntesis de nuestro trabajo de investigación, estos fueron los resultados:

\begin{tabular}{|l|l|}
\hline \multicolumn{1}{|c|}{ Julian Assange hasta 2012} & \multicolumn{1}{c|}{ Julian Assange después de 2012} \\
\hline Activista & Hacker \\
\hline Acusado de violación & Presunto violador \\
\hline (Víctima de) Conspiración, cerco y caza de brujas & Conspira (contra EE.UU) \\
\hline Divulga (información) & Roba (información) \\
\hline Marginado & Peligroso \\
\hline Presionado & Ataca \\
\hline Silenciado, amenazado & (Es para la sociedad un) Riesgo, amenaza \\
\hline
\end{tabular}

Tabla 2. Julian Assange después de pedir asilo a Ecuador

Fuente: Elaboración propia

\subsection{Julian Assange en la actualidad}

Esta es la parte del estudio que dedicamos a realizar un breve análisis de qué dicen del hacker que antaño inundaba portadas. Aunque cuando casi destrozas a la superpotencia que mueve el mundo es imposible que se olviden de ti, sí es cierto que las modas son pasajeras y que los medios de comunicación ayudan a que algunas causas queden enterradas.

El pasado mes de mayo (Junio 2017), El País publicó varias noticias sobre el herético cibernético australiano, todas en el mismo día. Analizamos las tres, sin embargo, hemos querido echar la vista un poco atrás, cuando en marzo de 2017 Assange asomó la cabeza por el gran ventanal que es Internet para criticar a la CIA y filtrar cierta información sobre ciberarmas. El País, en forma de noticia objetiva, dejaba ver una información con un claro tinte ideológico que resumimos en la siguiente tabla:

\begin{tabular}{|l|l|}
\hline \multicolumn{1}{|c|}{ Julian Assange } & \multicolumn{1}{c|}{ CIA } \\
\hline Filtra & Le filtran sus documentos \\
\hline Aprovecha (la débil situación de la CIA) & Se aprovechan de ella \\
\hline Se burla & Es burlada \\
\hline Ataca & Es atacada \\
\hline Ente activo & Ente pasivo \\
\hline
\end{tabular}

Tabla 3. Julian Assange Vs CIA (datos noticia 09/03/2017, El País) Fuente: Elaboración propia.

Como adelantábamos en párrafos anteriores, durante el estudio relativo a la actualidad de la cobertura mediática de El País sobre Julian Assange hemos realizado un análisis a tres noticias que aparecieron en la versión digital de El País el pasado 19 de mayo (2017). De las tres noticias analizadas solo una es "noticia real", en tanto que cumple con las dos premisas fundamentales del periodismo: es de actualidad y es relevante para la sociedad. 


\begin{tabular}{|c|c|c|}
\hline$\overline{N o t i c i a}$ & Sección & Síntesis \\
\hline $\begin{array}{l}\text { "Es inexplicable que Assange } \\
\text { mantenga la cordura" } \\
11: 21 \mathrm{~h}\end{array}$ & $\begin{array}{l}\text { TELEVISIÓN } \\
\text { NOTA: Sección poco } \\
\text { visitada y de escasa } \\
\text { importancia. }\end{array}$ & $\begin{array}{l}\text { Información sobre un documental acerca de } \\
\text { Julian Assange. Básicamente, la "noticia" se } \\
\text { limita a resumir, con citas y declaraciones de } \\
\text { los involucrados en el rodaje, el documental } \\
\text { en sí. }\end{array}$ \\
\hline $\begin{array}{l}\text { Wikileaks pese a Julian Assange } \\
17: 46 \mathrm{~h}\end{array}$ & INTERNACIONAL & $\begin{array}{l}\text { No es de actualidad, no aporta nada nuevo, } \\
\text { solo habla sobre la supuesta } \\
\text { arrogante y dictatorial personalidad de } \\
\text { Assange. }\end{array}$ \\
\hline $\begin{array}{l}\text { Suecia cierra la causa por } \\
\text { violación contra Julian Assange, } \\
\text { el fundador de Wikileaks } \\
20: 49 \mathrm{~h}\end{array}$ & INTERNACIONAL & $\begin{array}{l}\text { La verdadera noticia de actualidad, dada } \\
\text { después de habernos ofrecido información } \\
\text { sobre su talante arrogante y su condición de } \\
\text { soledad con respecto al grupo de } \\
\text { ciberactivistas, Wikileaks. }\end{array}$ \\
\hline
\end{tabular}

Tabla 4. Las noticias de actualidad sobre Julian Assange, titulares y trato informativo Fuente: Elaboración propia

\begin{tabular}{|c|}
\hline Hacker $(19 / 05 / 2017)$ \\
\hline Pirata $(19 / 05 / 2017)$ \\
\hline Polémico $(19 / 05 / 2017)$ \\
\hline
\end{tabular}

Tabla 5. Perfil de Julian Assange, según las noticias analizadas (datos textuales) Fuente: Elaboración propia

\section{El enfoque neurocientífico aplicado al Caso Assange}

Autores como Moodie, Studdert-Kennedy en Opiniones, públicos y grupos de presión o el mismo Young en La opinión pública y la propaganda definen a la opinión como "una creencia bastante fuerte o más intensa que una mera noción o impresión, pero menos que un conocimiento positivo basado sobre pruebas completas o adecuadas" (1982:10), es decir, algo alejado de una idea de convicción y de sentimiento que llaman "creencias emocionales", cuestión que podemos ampliar, de algún modo, sustentándonos en las teorías neurocientíficas que atribuyen al modo de trabajar la información que tiene el hipocampo, la forma en que finalmente actúa el individuo y las decisiones que este toma.

Y es que habríamos de destacar que, entre víctimas y culpables, o culpables y victimistas, o culpables y culpables, la verdadera base de todo este castillo de naipes es la propia biología humana. La neurociencia: el cerebro. Científicos como Xaro Sánchez, integrante de Investigación Estudios Conflictología, demuestran cómo nuestro cerebro está preparado para desarrollar prejuicios hacia los otros que son diferentes a nosotros, "son sesgos cognitivos, vías rápidas que tienen nuestros cerebros para desarrollar información, por eso se generan conflictos" (Redes (26/12/2010), RTVE:

http://www.rtve.es/alacarta/videos/redes/redes- cerebro-no-busca-verdad-sinosobrevivir/974036/). Esto quiere decir que la parte emocional de nuestro cerebro, o sistema 
límbico, se antepone a la razón, de modo que si hacemos uso del mismo, algunos argumentos que en frío nos resultarían perversos e inhumanos, dejando a un lado el raciocinio los concebimos como principios irrefutables.

Esto es lo que ocurre cuando los medios de comunicación nos crean sentimientos de antipatía o simpatía mediante el bombardeo de información y la segmentación de bandos, haciendo uso de las sensaciones que sirven de emoción directa a nuestro sistema límbico para llegar al objetivo primordial: la creación -y aceptación social- de un enemigo y un héroe comunes; Julian Assange Vs EEUU.

\section{El Análisis Crítico del Discurso en nuestra investigación}

Según Van Dijk, entre los objetos de dicha escuela o especialidad está la de repartir el poder entre aquellos que, en una sociedad jerárquica como esta, carecen de él, en palabras del lingüista Van Dijk, "ampliar el marco de la justicia y de la igualdad sociales." Esto quiere decir que, de cara a una sociedad mal informada por asuntos de Estructura de Información, al menos los ciudadanos habrían de saber defenderse con una formación cognitiva y psicológica más fuerte. Pongamos el caso que aquí analizamos del australiano Julian Assange. El País, de acuerdo con nuestro análisis que prosigue, se mostrará a favor o en contra según dicte el Poder: primero a favor, luego en contra. Lo primero porque aunque al Poder jamás vaya a resultarle conveniente una figura como tal, sí es cierto que nunca está de más que algunos medios jueguen al progresismo y hagan ver a la sociedad lo que denominaríamos un espejismo democrático que desaparece si te acercas para mirar con lupa.

\subsection{Poder y control}

La teoría principal de esta concepción es que a más poder tengas, con más habilidad, capacidad y oportunidad cuentas para controlar en función de tu interés propio. Esto es trasladable a los diferentes grupos sociales de una sociedad estratificada como esta.

Hallamos de entrada entonces, en nuestro análisis de las relaciones entre el discurso y el poder, que el acceso a formas específicas de discurso, p.e. las de la política, los media o la ciencia, es en sí mismo un recurso de poder. En segundo lugar, como hemos sugerido antes, nuestras mentes controlan nuestra acción; luego si somos capaces de influenciar la mentalidad de la gente, p.e. sus conocimientos o sus opiniones, podemos controlar indirectamente (algunas de) sus acciones. Y, en tercer lugar, puesto que las mentes de la gente son influidas sobre todo por los textos y por el habla, descubrimos que el discurso puede controlar, al menos indirectamente, las acciones de la gente, tal y como sabemos por la persuasión y la manipulación.

En definitiva, si un grupo tiene poder en demasía, podrá además controlar las mentes de las personas que no tengan ese poder. De ahí el objetivo del ACD del que se hablaba con anterioridad acerca de repartir el poder, para lo cual, por cierto, haría falta mejorar no solo la capacidad crítica de la sociedad, sino su capacidad cognitiva y el deseo de saber. El ciudadano que compra su periódico o consume informativos de radio o televisión p.e. para saber sobre Julian Assange ha de mostrarse no cínico ni incrédulo, pero sí distante y preparado para con la información que a este le llega, y que viene directa o indirectamente proporcionada por el Poder en forma de redactores, reporteros o columnistas. 
$Y$ es que hablar en ocasiones se torna sinónimo de manipular, y viceversa. En un mundo donde la oratoria es a la vez necesidad y arma; hermandad y ataque, el poder se disfraza a veces de habla (recordemos las palabras de Orfeo/Unamuno).

\section{Conclusiones}

Como conclusión, pensamos que efectivamente Julian Assange ha sufrido una metamorfosis en cuanto a consideración social, en tanto que primero ha sido admirado como un individuo bueno y respetable, y después todo lo contrario. Así mismo, tras nuestro estudio analítico y crítico de los artículos periodísticos escogidos, nos reafirmamos en la postura mostrada en nuestras hipótesis al considerar que fue el cobijo del entonces presidente de Ecuador, Rafael Correa, uno de los mayores puntos de inflexión en la metamorfosis del australiano: de bueno a malo en cuestión de un año. Pura simplificación del mensaje en el periodismo mediante la dinámica dualista.

De igual modo, estimamos que es EEUU aquel que piensa y decide por todos, marcando el rumbo del periódico y sus redactores, a fin de que los lectores cosechen la idea que quiera sembrar esta superpotencia: una propaganda libre de críticas e inestabilidades.

Los primeros 291 de los 251.287 documentos obtenidos por WikiLeaks fueron difundidos el 28 de noviembre desde el servidor de la organización y de manera simultánea a una detallada cobertura de prensa de los diarios El País (España), Le Monde (Francia), Der Spiegel (Alemania), TheGuardian (Reino Unido) y The New York Times (Estados Unidos). Sería interesante continuar con una investigación que profundice sobre la cobertura que estos medios ofertaban a la sociedad y analizar la orquestación existente entre ellos.

Así mismo, consideramos que realizar tal trabajo centrándose especialmente desde el punto de vista de la Estructura de la Información, enriquecería el trasfondo del mismo y daría muchas respuestas a las preguntas que se nos pueden plantear al observar cierto comportamiento simbiótico entre estos medios, y que solo dicha disciplina puede responder.

\section{Anexo}

\section{Artículos analizados}

Alandete, D. Monge, Y. (02/12/2010). Objetivo: Desenchufar completamente a Wikileaks. El País. Recuperado de https://elpais.com/internacional/2010/12/02/actualidad/1291244440_850215.ht ml Alandete, D. Monge, Y. (10/12/2010). Los abogados de Assange temen una acusación de EE UU por espionaje. EL País. Recuperado de https://elpais.com/internacional/2010/12/10/actualidad/1291935618_850215.ht ml

Alandete, D. (16/08/2012). EE UU niega haber ejercido presión sobre Londres para que extradite a Assange. El País. Recuperado de https://elpais.com/internacional/2012/08/16/actualidad/1345142621_831034.ht ml

Alandete, D. (16/08/2012). EE UU no ha acusado ni ha pedido la extradición de Assange. El País. Recuperado de https://elpais.com/internacional/2012/08/16/actualidad/1345124436_205345.ht ml

Barbieri, P. (04/07/2013). Las dos Latinoaméricas se alejan. El País. Recuperado de https://elpais.com/elpais/2013/06/28/opinion/1372443957_660252.html

Basterra, F. G. (10/12/2010). Prohibido prohibir. El País. Recuperado de https://elpais.com/internacional/2010/12/10/actualidad/1291935627_850215.ht ml 
EFE (21/08/2012). Anonymous ataca la web de Justicia británica. El País. Recuperado de https://elpais.com/tecnologia/2012/08/21/actualidad/1345543962_517735.html

Elola, J. (18/12/2010). "Wikileaks sufre una caza de brujas”. El País. Recuperado de https://elpais.com/elpais/2010/12/18/actualidad/1292663831_850215.html

El País. (03/12/2010). “¿Sobreviviremos? Eso depende de ti”. El País. Recuperado de https://elpais.com/internacional/2010/12/03/actualidad/1291330812_850215.ht ml

El País. (04/12/2010). PayPal desactiva la cuenta de WikiLeaks. El País. Recuperado de https://elpais.com/internacional/2010/12/04/actualidad/1291417204_850215.ht ml

El País. (09/12/2010). Facebook cierra la cuenta del grupo de ciberactivistas defensores de Wikileaks. El País. Recuperado de https://elpais.com/internacional/2010/12/09/actualidad/1291849211_850215.ht $\mathrm{ml}$

El País. (13/12/2010). Las páginas europeas de Amazon caen durante media hora. El País. Recuperado de https://elpais.com/internacional/2010/12/13/actualidad/1292194805_850215.ht ml

El País. (16/12/2010). EE UU intenta armar un caso de conspiración contra Assange. El País. Recuperado de https://elpais.com/internacional/2010/12/16/actualidad/1292454002_850215.ht ml

El País. (20/08/2012). La prensa británica y ecuatoriana critican la estrategia de Julian Assange. EI País. Recuperado de https://elpais.com/internacional/2012/08/20/actualidad/1345467247_643937.ht $\mathrm{ml}$

Garín, J. (23/04/2013). La extraña entrevista de Ana Ibáñez a Correa. Rebelión. Recuperado de http://www.rebelion.org/noticia.php?id=167125

González, M. (31/05/2013). Assange, riesgo para la seguridad. El País. Recuperado de https://politica.elpais.com/politica/2013/05/31/actualidad/1370028224_937330.h tml

Gutiérrez, O. (19/05/2017). Wikileaks pese a Julian Assange. El País. Recuperado de https://elpais.com/internacional/2017/05/19/actualidad/1495200002_237259.ht ml

Jiménez, V. (28/11/2010). La mayor filtración de la historia deja al descubierto los secretos de la política exterior de EE UU. El País. Recuperado de https://elpais.com/internacional/2010/11/28/actualidad/1290898825_850215.ht ml

Junquera, N. (19/05/2017). "Es inexplicable que Assange mantenga la cordura". El País. Recuperado de https://elpais.com/cultura/2017/05/18/television/1495102583_179047.html

Junquera, N. Guimón, P. (19/05/2017). Suecia cierra la causa por violación contra Julian Assange, el fundador de Wikileaks. El País. Recuperado de https://elpais.com/internacional/2017/05/19/actualidad/1495184926_238900.ht ml

Lozano, J. Francescutti, P. (07/12/2012). Cuando desvelar es también ocultar. El País. Recuperado de https://elpais.com/elpais/2012/09/21/opinion/1348253243_578874.html

Martínez, J. (09/03/2017). Assange: "La CIA ha perdido el control de su arsenal de armas cibernéticas". El País. Recuperado de https://elpais.com/internacional/2017/03/09/estados_unidos/1489072157_1439 83.html

Monge, Y. Alandete, D. (01/12/2010). Estados Unidos estrecha el cerco sobre Wikileaks. El País. Recuperado de https://elpais.com/internacional/2010/12/01/actualidad/1291158036_850215.ht ml

Naím, M. (29/06/2013). Rafael Correa, paladín de la libertad. El País. Recuperado de https://elpais.com/internacional/2013/06/29/actualidad/1372530577_320933.ht ml

Otero, B. (05/09/2012). Assange se queda sin contactos en su agenda VIP. El País. Recuperado de https://elpais.com/elpais/2012/09/04/gente/1346778729_621249.html

Palacio, A. (22/08/2012). Julian Assange: fraude al Estado de derecho. El País. Recuperado de https://elpais.com/elpais/2012/08/21/opinion/1345551622_753165.html

Prados, L. (05/12/2011). "Wikileaks puso sobre la mesa la relación entre prensa y poder". El País. Recuperado de https://elpais.com/sociedad/2011/12/07/actualidad/1323258404_591211.html 
Rebossio, A. (10/07/2013). Los países latinoamericanos reaccionan a su manera ante el espionaje de EE UU. El País. Recuperado de

https://elpais.com/internacional/2013/07/10/actualidad/1373477708_070754.ht ml

Tubella, P. (04/12/2010). "Ni un solo individuo ha sido perjudicado por nuestras filtraciones". El País. Recuperado de https://elpais.com/diario/2010/12/04/internacional/1291417207_850215.html

Tubella, P. (19/08/2012). Assange pide a Obama que renuncie a la "caza de brujas" contra Wikileaks. El País. Recuperado de https://elpais.com/internacional/2012/08/19/actualidad/1345383033_632725.ht $\mathrm{ml}$

\section{Referencias}

Freud, S. (1979). El porvenir de una ilusión. Madrid, España: Alianza Editorial.

Freud, S. (1979). Más allá del principio del placer. Madrid, España: Alianza Editorial.

Freud, S. (1979). Psicología de las masas. Madrid, España: Alianza Editorial.

Labio Bernal, A. (2006). Comunicación, periodismo y control informativo. Barcelona, España: Anthropos Editorial.

Maslow, A. (1943). A Theory of Human Motivation, en emotionalliteracyeducation.

Moodie, G. C. Studdert-Kennedy, G. (1975). Opiniones, públicos y grupos de presión.

Núñez Domínguez, T. (Coord.a). (2014). Competencias psicosociales para profesionales de los medios. Madrid, España: Ediciones Pirámide (Grupo Anaya).

Reig, R. (2015). Crisis del sistema, crisis del periodismo. Barcelona, España: Editorial Gedisa

Reig, R. (2004). Dioses y diablos mediáticos. Barcelona, España: Ediciones Urano.

Reig, R. (2000). La telaraña mediática. Zamora, España: Comunicación Social Ediciones y Publicaciones.

Reig, R. (2011). Los dueños del periodismo. Barcelona, España: Editorial Gedisa.

Rendueles, C. (2013). Sociofobia: El cambio político en la era de la utopía digital, España: Capitán Swing.

Sierra Caballero, F. (1999). Elementos de Teoría de la Información. Alcalá de Guadaira, España: Editorial Mad.

Young, K. (1986). La opinión pública y la propaganda. México, D.F.: Editorial Electrocomp.

\section{BREVE SEMBLANZA DE LA AUTORA:}

Helena Ramírez Barragán es Graduada en Periodismo por la Universidad de Sevilla, Fotógrafa profesional, colabora con el Laboratorio de Estudios en Comunicación (LADECOM). 
- Recibido: 21/01/2018

- Aceptado: 02/02/2018

Ámbitos. Revista Internacional de Comunicación, n.40, edición de primavera, 2018. 